\title{
Turbulence in More than Two and Less than Three Dimensions
}

\author{
Antonio Celani, ${ }^{1}$ Stefano Musacchio, ${ }^{2,3}$ and Dario Vincenzi ${ }^{3}$ \\ ${ }^{1}$ CNRS URA 2171, Institut Pasteur, 28 rue du docteur Roux, 75724 Paris Cedex 15, France \\ ${ }^{2}$ Dipartimento di Fisica Generale and INFN, Università di Torino, via P. Giuria 1, 10125 Torino, Italy \\ ${ }^{3}$ CNRS UMR 6621, Laboratoire J. A. Dieudonné, Université de Nice Sophia Antipolis, Parc Valrose, 06108 Nice, France
}

(Received 12 January 2010; published 7 May 2010)

\begin{abstract}
We investigate the behavior of turbulent systems in geometries with one compactified dimension. A novel phenomenological scenario dominated by the splitting of the turbulent cascade emerges both from the theoretical analysis of passive scalar turbulence and from direct numerical simulations of NavierStokes turbulence.
\end{abstract}

DOI: 10.1103/PhysRevLett.104.184506

PACS numbers: 47.27.Ak, 47.27.ek, 47.27.T-

In statistical physics most systems display a strong dependence on the space dimensionality. The best known example is the existence of critical dimensions in phase transitions. Among far-from-equilibrium systems, hydrodynamic turbulence shows a remarkable dependence on the spatial dimension as well. In three dimensions, the nonlinear interaction between different scales is described by the Kolmorogov-Richardson direct cascade: the kinetic energy injected at large scale by an external forcing is transferred to smaller and smaller eddies until it reaches the scales where it is dissipated by viscosity [1]. By contrast, in two dimensions, the simultaneous conservation of kinetic energy and enstrophy results in an inverse energy cascade; i.e., the energy injected by the forcing is transferred to large-scale structures [2]. Moreover, threedimensional turbulence is characterized by anomalous scaling and small-scale intermittency [1], whereas the inverse cascade is apparently self-similar and even shows some signatures of conformal invariance [3]. The transition between the two behaviors and the possible existence of a critical dimension between $d=3$ and $d=2$ have been investigated mainly in models of turbulence where the dimension was introduced as a formal parameter [4-6]. In this Letter, we rather opt for a geometrical way of looking in between integer dimensions, and examine turbulent systems which can be regarded as transitional between a $d$-dimensional isotropic system and a $(d-1)$ dimensional one. Namely, we consider a $d$-dimensional isotropic system and make one dimension of the space periodic. The compactified dimension can then be collapsed or inflated at will so as to connect continuously the two extreme cases. We start by considering the turbulent transport of a passive scalar field. This system displays a close similarity to hydrodynamic turbulence (intermittent direct cascade of scalar variance or scale-invariant inverse cascade depending on the properties of the flow), and has the advantage of being analytically solvable [7]. We then study the full hydrodynamical problem by means of direct numerical simulations. The novel feature that emerges from our study is the splitting of the turbulent cascade in both systems. The scalar variance (or the kinetic energy) injected at a given length scale flows both toward small and large scales, thus giving rise to a simultaneous double cascade of the same quantity.

Let us start with the analysis of the analytically solvable case. The evolution of a passive scalar field $\theta(\mathbf{x}, t)$ is described by the advection-diffusion equation:

$$
\partial_{t} \theta+\mathbf{v} \cdot \nabla \theta=\kappa \nabla^{2} \theta+\varphi,
$$

where $\kappa$ is the diffusivity of the scalar and $\varphi(\mathbf{x}, t)$ is the source term. The scalar field does not influence the velocity, whose statistical properties are given. We model the turbulent flow by means of the Kraichnan ensemble [8]. Thus, $\mathbf{v}(\mathbf{x}, t)$ is a Gaussian stochastic process with zero mean and correlation: $\left\langle v_{\alpha}(\mathbf{x}+\mathbf{r}, t+\tau) v_{\beta}(\mathbf{x}, t)\right\rangle=$ $\left[d_{\alpha \beta}^{0}-d_{\alpha \beta}(\mathbf{r})\right] \delta(\tau)$ with $d_{\alpha \beta}^{0}$ constant and $d_{\alpha \beta}(-\mathbf{r})=$ $d_{\alpha \beta}(\mathbf{r})$. The flow is statistically homogeneous, parity invariant, stationary, and invariant under time reversal. The tensor $2 d_{\alpha \beta}(\mathbf{r})$ represents the spatial correlation of the velocity differences [7]; its form will be specified later.

The source is assumed to be random as well, and more specifically Gaussian, independent of the velocity, with zero mean and correlation: $\langle\varphi(\mathbf{x}+\mathbf{r}, t+\tau) \varphi(\mathbf{x}, t)\rangle=$ $\Phi(\mathbf{r}) \delta(\tau)$, where $\Phi(\mathbf{r})$ rapidly decays to zero for $r=\|\mathbf{r}\|$ greater than the correlation length $\ell_{\varphi}$.

To study the cascade of scalar variance, we consider the single-time correlation $\quad C(\mathbf{r}, t) \equiv\langle\theta(\mathbf{x}+\mathbf{r}, t)) \theta(\mathbf{x}, t)\rangle$, which does not depend on $\mathbf{x}$ owing to the statistical homogeneity of the velocity. In the Kraichnan model, $C(\mathbf{r}, t)$ satisfies the partial differential equation

$$
\partial_{t} C=\mathcal{M}(\mathbf{r}) C+\Phi,
$$

where $\mathcal{M}(\mathbf{r})=d_{\alpha \beta}(\mathbf{r}) \partial_{r_{\alpha}} \partial_{r_{\beta}}+2 \kappa \partial_{r_{\alpha}} \partial_{r_{\alpha}}$ (summation over repeated indexes is implied) [8]. Without loss of generality, we assume $\theta(\mathbf{x}, 0)=0$ and hence $C(\mathbf{r}, 0)=0$. The (generalized) solution of Eq. (2) then takes the form [9]

$$
C(\mathbf{r}, t)=\int_{0}^{t} d s \int d \boldsymbol{\rho} \Phi(\boldsymbol{\rho}) p(\mathbf{r}, t ; \boldsymbol{\rho}, s) .
$$

In the above equation, $p(\mathbf{r}, t ; \boldsymbol{\rho}, s)$ is the probability density function that two fluid particles being at separation $\mathbf{r}$ at 
time $t$ were at separation $\boldsymbol{\rho}$ at time $s<t$. The probability density function describes the backward-in-time separation of Lagrangian trajectories and satisfies

$$
-\partial_{s} p(\mathbf{r}, t ; \boldsymbol{\rho}, s)=\mathcal{M}^{\dagger}(\boldsymbol{\rho}) p(\mathbf{r}, t ; \boldsymbol{\rho}, s),
$$

where $\mathcal{M}^{\dagger}$ denotes the adjoint of $\mathcal{M}: \mathcal{M}^{\dagger}(\boldsymbol{\rho})=$ $\partial_{\rho_{\alpha}} \partial_{\rho_{\beta}} d_{\alpha \beta}(\boldsymbol{\rho})+2 \kappa \partial_{\rho_{\alpha}} \partial_{\rho_{\alpha}}$. Eqs. (2) to (4) establish the link between the Eulerian statistics of the scalar field and the Lagrangian dynamics of fluid particles [7].

Gawędzki and Vergassola have studied a $d$-dimensional version of the Kraichnan model where the flow is in addition statistically isotropic and compressible and where $d_{\alpha \beta}(\mathbf{r})$ is scale invariant with exponent $0<\xi<2$ [10]. This latter property implies that, in a typical realization, $\mathbf{v}(\mathbf{x}, t)$ is not smooth in space and its spatial regularity decreases with decreasing $\xi$. The compressibility degree of the flow is defined as $\wp=\left\langle(\nabla \cdot \mathbf{v})^{2}\right\rangle /\left\langle\operatorname{tr}(\nabla \mathbf{v})^{2}\right\rangle$ and satisfies $0 \leq \wp \leq 1$. In this model, the Reynolds number is formally infinite since there is neither a viscous nor a largescale regularization of the velocity.

To understand passive scalar turbulence in geometries with a compactified dimension, it is instructive to briefly review the two- and one-dimensional isotropic cases under the assumptions of Ref. [10]. We shall directly consider the limit $\kappa \rightarrow 0$.

On the Euclidean plane, three regimes can be identified [10]. For weak compressibility $(\wp<1 / 2)$, Lagrangian trajectories separate superdiffusively, thus generating a smallscale dissipation of scalar fluctuations. Correspondingly, the scalar field displays a stationary direct cascade of $\left\langle\theta^{2}\right\rangle$; i.e., the variance injected by the source is entirely transferred to small scales and eventually dissipated at a constant rate $\epsilon_{\text {down }}$ equal to the injection rate $\Phi(0)$. In this regime, Eq. (2) has a time-independent solution and $\left\langle\theta^{2}(t)\right\rangle=C(\mathbf{0}, t)$ converges in time to a constant value. For strongly compressible flows $\left(\wp \geq 2 / \xi^{2}\right)$, Lagrangian trajectories rather tend to collapse in time, and thus transfer scalar variance to large-scale structures in an inversecascade process. In terms of the above Lagrangian formulation of transport [Eqs. (2) to (4)], the inverse cascade of $\left\langle\theta^{2}\right\rangle$ follows from the convergence of $p(\mathbf{r}, t ; \boldsymbol{\rho}, s)$ to $\delta(\boldsymbol{\rho})$ as $|t-s| \rightarrow \infty$. Inserting the long-time behavior of $p(\mathbf{r}, t ; \boldsymbol{\rho}, s)$ into Eq. (3) indeed yields: $\left\langle\theta^{2}(t)\right\rangle \sim \epsilon_{\text {up }} t$ as $t \rightarrow$ $\infty$ with $\epsilon_{\text {up }}=\Phi(0)$. Finally, in the regime of intermediate compressibility $\left(1 / 2<\wp<2 / \xi^{2}\right)$, the long-time behavior of the variance is $\left\langle\theta^{2}(t)\right\rangle=O\left(t^{b}\right)$ with $0<b<1$. The scalar variance is partly dissipated at small scales at a rate that equals the injection rate only for $t \rightarrow \infty$. Simultaneously, a fraction of $\left\langle\theta^{2}\right\rangle$ is transferred to large scales at a rate asymptotically vanishing in time.

On the real line, the only relevant parameter is $\xi$. The value $\xi=1$ marks a phase transition in the dynamics of fluid particles and hence in the behavior of the scalar [10]. For $\xi>1$, the trajectories collapse in time and produce an inverse cascade of $\langle\theta\rangle^{2}$ with transfer rate $\epsilon_{\text {up }}=\Phi(0)$. For $\xi<1,\left\langle\theta^{2}(t)\right\rangle=O\left(t^{1 /(2-\xi)}\right)$ so that $\epsilon_{\text {down }}$ equals $\Phi(0)$ only in the limit $t \rightarrow \infty$, as in the regime of intermediate compressibility in two dimensions.

As a transitional manifold between the plane and the straight line, we consider the surface of an infinite cylinder, which can be inflated or collapsed to reproduce the two limiting geometries. A cylindrical surface is described by two coordinates: $-\infty<r_{1}<\infty$ and $-\pi L \leq r_{2}<\pi L$, where $L$ is the radius of the cylinder. The isotropic Kraichnan ensemble can be generalized to this geometry by defining $d_{\alpha \beta}(\mathbf{r})=\sum_{k_{2} \in(1 / L) \mathbb{Z}} \int_{-\infty}^{\infty} d k_{1}\left(1-e^{i \mathbf{k} \cdot \mathbf{r}}\right) F_{\alpha \beta}(\mathbf{k})$, where the sum is taken over integer multiples of $1 / L$ and

$$
F_{\alpha \beta}(\mathbf{k}) \propto k^{-2-\xi}\left[(1-\wp) \delta_{\alpha \beta}+(2 \wp-1) k^{-2} k_{\alpha} k_{\beta}\right] .
$$

The Fourier series in $d_{\alpha \beta}(\mathbf{r})$ guarantees the periodicity of $\mathbf{v}(\mathbf{x}, t)$ with respect to the radial coordinate $r_{2}$. With this definition, the limit of $d_{\alpha \beta}(\mathbf{r})$ for $L \rightarrow \infty$ yields the isotropic Kraichnan ensemble on the plane with degree of compressibility $\wp$ and scaling exponent $\xi[7,10,11]$. We take $\wp<1 / 2$ so that the limiting system exhibits a stationary direct cascade of $\left\langle\theta^{2}\right\rangle$. The opposite limit, $L \rightarrow 0$, yields the one-dimensional Kraichnan ensemble with scaling exponent $\xi+1$, where $\xi$ must be taken less than one so that $\xi+1<2[7,10,11]$. According to the above discussion of the isotropic case, the limit of vanishing radius leads to the inverse cascade of $\left\langle\theta^{2}\right\rangle$. By varying $L$, it is thus possible to move from a direct cascade in two dimensions to an inverse cascade in one dimension.

For any $0<L<\infty$, a qualitative picture of the dynamics can be inferred from the one- and two-dimensional isotropic cases. We assume for instance $\ell_{\varphi}>L$. At $r \ll$ $L$, fluid particles move in a two-dimensional isotropic flow with $\wp<1 / 2$; therefore, they separate superdiffusively and dissipate $\left\langle\theta^{2}\right\rangle$ at a constant rate. At $r \gg L$, fluid particles move in an essentially one-dimensional flow with scaling exponent larger than 1; they tend to collapse and thus transfer scalar variance to scales larger than $\ell_{\varphi}$. As a consequence, the cascade of $\left\langle\theta^{2}\right\rangle$ splits into a direct branch and an inverse one. In this system, small-scale dissipation and transfer to large scales coexist. Similar arguments can be repeated for $\ell_{\varphi}<L$.

A quantitative prediction of the split-cascade process can be obtained through an approximate analysis of Eq. (4). We first consider the case $\ell_{\varphi} \gg L$. At separations $\rho \gg L$, the components of $d_{\alpha \beta}(\boldsymbol{\rho})$ can be replaced with their asymptotic expansions: $d_{11}(\boldsymbol{\rho}) \sim D_{1}\left|\rho_{1}\right|^{1+\xi}+\tilde{\kappa}_{1}$, $d_{22}(\boldsymbol{\rho}) \sim D_{2}\left|\rho_{1}\right|^{1+\xi}+\tilde{\kappa}_{2}$, and $d_{12}(\boldsymbol{\rho})=d_{21}(\boldsymbol{\rho}) \sim 0$ with $D_{i}$ and $\tilde{\kappa}_{i}$ positive constants $(i=1,2)$ [11]. The expansion of $d_{\alpha \beta}(\boldsymbol{\rho})$ shows that, at large separations, the velocity difference is the sum of two independent one-dimensional random fields. In particular, the axial component is a Kraichnan flow with scaling exponent $\xi+1$. At $\rho \gg L$, $d_{11}(\boldsymbol{\rho})$ and $d_{22}(\boldsymbol{\rho})$ include the effective diffusivities $\tilde{\boldsymbol{\kappa}}_{1}$ and $\tilde{\kappa}_{2}$, which are reminiscence of the small-scale motion. We can therefore set $\kappa=0$ in the present analysis as well. We consider $p_{1}\left(\mathbf{r}, t ; \rho_{1}, s\right)=\int_{-\pi L}^{\pi L} d \rho_{2} p\left(\mathbf{r}, t ; \rho_{1}, \rho_{2}, s\right)$. Integrating Eq. (4) over $\rho_{2}$ and taking into account periodic 
boundary conditions, $\left[\partial_{\rho_{2}} p\right]_{\rho_{2}=\pi L}=\left[\partial_{\rho_{2}} p\right]_{\rho_{2}=-\pi L}$, yields a partial differential equation for $p_{1}\left(\mathbf{r}, t ; \rho_{1}, s\right)$. This equation admits the time-independent solution $p_{1}\left(\mathbf{r}, t ; \rho_{1}, s\right) \propto$ $\left(D_{1}\left|\rho_{1}\right|^{1+\xi}+\tilde{\kappa}_{1}\right)^{-1}$, which we can insert in Eq. (3) to compute the long-time behavior of $\left\langle\theta^{2}(t)\right\rangle$. For the sake of simplicity, we further assume $\Phi(\mathbf{r})=\Phi(0)$ if $r \leq \ell_{\varphi}$ and zero otherwise. Then, Eq. (3) yields: $\Phi(0) t-$ $C\left(t, r_{1}\right) \propto \Phi(0)\left(L / \ell_{\varphi}\right)^{\xi} t$, so that a fraction $\propto\left(L / \ell_{\varphi}\right)^{\xi}$ of the injected variance does not flow upwards, and is eventually dissipated at small scales. If $S(\mathbf{r})=\langle[\theta(\mathbf{x}+\mathbf{r}, t)-$ $\left.\theta(\mathbf{x}, t)]^{2}\right\rangle$ denotes the scalar structure function, the above behavior is consistent with the picture of an inverse cascade with $S\left(\left|r_{1}\right|\right) \sim\left(\Phi(0) / D_{1}\right)\left\{1-O\left[\left(L / \ell_{\varphi}\right)^{\xi}\right]\right\}\left|r_{1}\right|^{1-\xi}$ at $\left|r_{1}\right| \gg \ell_{\varphi}$, along with a small-scale behavior $S\left(\left|r_{1}\right|\right) \sim$ $\left(\Phi(0) / D_{1}\right) \ell_{\varphi}^{-\xi}\left|r_{1}\right|-\left(\Phi(0) / D_{1}\right) O\left[\left(L / \ell_{\varphi}\right)^{\xi}\right]\left|r_{1}\right|^{1-\xi} \quad$ at $L \ll\left|r_{1}\right| \ll \ell_{\varphi}$. In the right-hand side of the latter expression, the second term is the inhomogeneous contribution arising from $\partial_{t} C-\Phi(0)$ and the first one is the zero mode needed to match the correlation function. The remaining direct cascade flux $\tilde{\kappa}_{1} S(L) / L^{2} \sim \Phi(0)\left(L / \ell_{\varphi}\right)^{\xi}$ is then transferred to two-dimensional motion at $r \lesssim L$ and finally dissipated at small scales.

The case $\ell_{\varphi} \ll L$ can be examined by computing the small-scale form of $p(\mathbf{r}, t ; \boldsymbol{\rho}, s)$. At scales $\rho \ll L, \mathcal{M}^{\dagger}(\boldsymbol{\rho})$ can be replaced with its two-dimensional isotropic form: $M^{\dagger}(\rho) \propto \rho^{-1} \partial_{\rho} \rho^{a} \partial_{\rho} \rho^{1+\xi-a}$ with $a=-1+(2+$ $\xi) /(\wp \xi+1)$ [10]. The zero mode of $M^{\dagger}(\rho)$ corresponding to zero flux at $\rho=0$ gives $p(\mathbf{r}, t ; \boldsymbol{\rho}, s) \propto \rho^{a-1-\xi}$ for $\rho \ll$ $L$. We can then insert this behavior in Eq. (3) to obtain: $\left\langle\theta^{2}(t)\right\rangle \sim \epsilon_{\text {up }} t$ as $t \rightarrow \infty$ with $\epsilon_{\text {up }} \propto \Phi(0)\left(\ell_{\varphi} / L\right)^{1+a-\xi}$. Once more, a fraction of the injected variance is transferred to large scales, whereas the remaining part is dissipated at small scales. The splitting of the cascade of scalar variance, shown here in the asymptotic cases $\ell_{\varphi} / L \gg 1$ and $\ell_{\varphi} / L \ll 1$, holds for a generic $\ell_{\varphi} / L$ as a result of the convergence of $p(\mathbf{r}, t ; \boldsymbol{\rho}, s)$ to a broad-in- $\boldsymbol{\rho}$ timeindependent form [11]. Combining this latter result with Eq. (3) indeed yields: $\left\langle\theta^{2}(t)\right\rangle \sim \epsilon_{\text {up }} t$ as $t \rightarrow \infty$ with $\epsilon_{\text {up }}<\Phi(0)$.

Some remarks conclude the analysis of passive transport on a cylindrical surface. First, the above results have been obtained for a Prandtl number $\operatorname{Pr} \equiv \nu / \kappa=0(\nu$ is the kinematic viscosity of the fluid). Provided that $\wp<1 / 2$, we nevertheless expect the cascade splitting for any $\operatorname{Pr}>0$ as well as in the limit $\operatorname{Pr} \rightarrow \infty$. The small-scale flow is indeed weakly compressible, and therefore nearby trajectories separate irrespective of the value of $\operatorname{Pr}$ [12]. Moreover, the large-scale strongly compressible nature of the flow does not depend on Pr. Second, varying $L$ from infinity to zero produces a smooth modification of the cascade; i.e., the dissipation rate $\epsilon_{\text {down }}$ continuously varies from $\Phi(0)$ to zero. The system, therefore, does not exhibit a phase transition as a function of $L$.

Let us now discuss the case of hydrodynamic turbulence. The dynamics of the velocity field $\mathbf{u}(\mathbf{x}, t)$ is given by the
Navier-Stokes equation

$$
\partial_{t} \mathbf{u}+\mathbf{u} \cdot \nabla \mathbf{u}=-\nabla P / \varrho+\nu \nabla^{2} \mathbf{u}+\mathbf{f}
$$

supplemented by the incompressibility constraint $\nabla \cdot \mathbf{u}=$ 0 . Here $P$ denotes the pressure and $\varrho$ is the density of the fluid. The flow is sustained by a stochastic Gaussian, whitein-time noise $\mathbf{f}(\mathbf{x}, t)$. The forcing is active only on the horizontal velocities $u_{x}, u_{y}$, it depends only on the horizontal components $x, y$, and it is localized in Fourier space in a narrow band of wave numbers $|\mathbf{k}| \simeq k_{f}$. Direct numerical simulations were performed by means of a standard 2/3-dealiased pseudospectral method on a periodic domain with various aspect ratios, as in Ref. [13]. This geometrical configuration can be considered as intermediate between two- and three-dimensional isotropic turbulence. The numerical simulations were conducted with uniform grid spacing, square basis $L_{x}=L_{y}$, aspect ratio $R=L_{x} / L_{z}$, and resolution $N_{x}=N_{y}=R N_{z}=1024$. Viscous dissipation has been replaced by an hyperviscous damping term $(-1)^{p-1} \nu_{p} \nabla^{2 p} \mathbf{u}$.

We studied the transition from $2 \mathrm{D}$ to $3 \mathrm{D}$ at varying the aspect ratio of the domain. The mean kinetic energy $E(t)=$ $1 / 2\left\langle|\mathbf{u}(\mathbf{x}, t)|^{2}\right\rangle$ provides a suitable probe of the transition. In $2 \mathrm{D}$ flows at high Reynolds number, kinetic energy grows linearly in time with a growth rate almost equal to the injection rate $\epsilon_{\text {in. }}$. Conversely, in 3D flows, after an initial transient $t_{0}$ required to build up the turbulent cascade, the kinetic energy attains a statistically constant value. In the intermediate situation realized in our setup, we observe a linear growth of kinetic energy for $t>t_{0}$ (see Fig. 1), as in the two-dimensional case, but the growth rate diminishes as the vertical scale $L_{z}$ is increased, and almost vanishes when $L_{z} / \ell_{f} \simeq 1 / 2$. As in the case of the scalar field, this behavior suggests that the turbulent cascade splits also in the hydrodynamic case. Within this picture, part of the energy injected by the forcing is transferred toward large

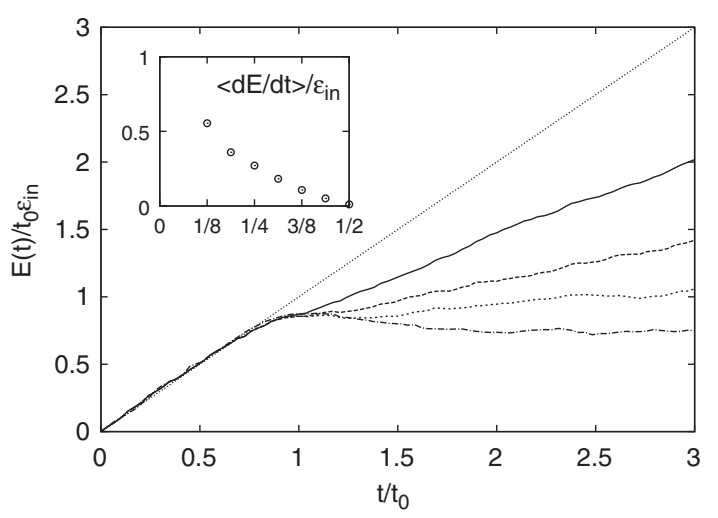

FIG. 1. Growth of mean kinetic energy for various values of the aspect ratio $L_{z} / \ell_{f}=1 / 8,1 / 4,3 / 8,1 / 2$ (from top to bottom). The straight line represents the theoretical growth rate for a two-dimensional flow. Inset: Growth rates $\langle d E / d t\rangle$ as a function of the ratio $L_{z} / \ell_{f}$. Parameters of the simulation: $L_{x}=2 \pi, \ell_{f} / L_{x}=1 / 16, \nu_{8}=10^{-38}$. 


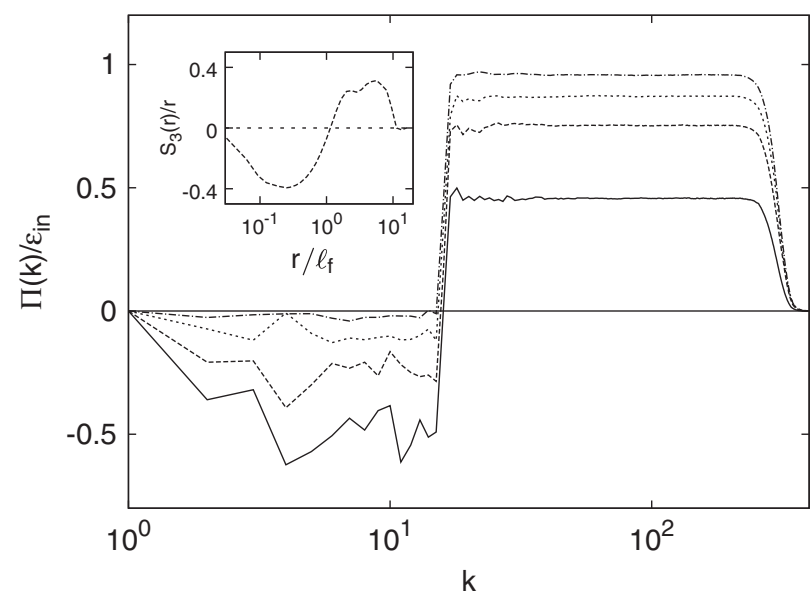

FIG. 2. Spectral flux of kinetic energy for various aspect ratio $L_{z} / \ell_{f}=1 / 8,1 / 4,3 / 8,1 / 2$ (from bottom to top). Simulation parameters as in Fig. 1. The inset reports the third order structure function of the velocity, $S_{3}(r)$, for $L_{z} / \ell_{f}=1 / 4$.

scales and feeds an inverse energy cascade as in twodimensional flows. The remnant energy gives rise to a direct energy cascade toward small scales, as in the three-dimensional case.

This suggestive scenario is supported by the inspection of the spectral fluxes of kinetic energy (see Fig. 2). The fluxes show a flat positive plateau in the wave-number range $k>k_{f}$, which confirms the presence of a direct cascade at small scale. At small wave number $k<k_{f}$, the fluxes have stronger fluctuations, but their mean negative value is consistent with the presence of an inverse energy cascade. As expected, the ratio between the energy flux $\epsilon_{\text {up }}$ and $\epsilon_{\text {down }}$ of the inverse and direct cascade, respectively, is a decreasing function of the ratio $L_{z} / \ell_{f}$.

In the range of scales corresponding to the inverse cascade the energy spectrum of horizontal velocities has a scaling region $E_{H}(k) \sim \epsilon_{\text {up }}^{2 / 3} k^{-5 / 3}$, as observed in twodimensional flows. At scales smaller than $L_{z}$, Kolmogorov spectra are observed both for horizontal and vertical velocities, signaling the presence of three-dimensional turbulence at small scales (see Fig. 3).

We argue that the cascade splitting described above, i.e., coexistence of 3D and 2D turbulence, should take place whenever the flow is confined in one direction, be it by material boundaries or by any other physical mechanism of dimensional reduction, e.g., stable stratification.

Whether or not Navier-Stokes turbulence displays a phase transition from this novel regime to the classical three-dimensional regime remains still unclear. Our numerical results seem to indicate that the inverse-cascade flux becomes negligible as the vertical scale approaches the forcing length-scale, therefore supporting the conjecture of a phase transition at some critical value of the ratio $L_{z} / \ell_{f}$.

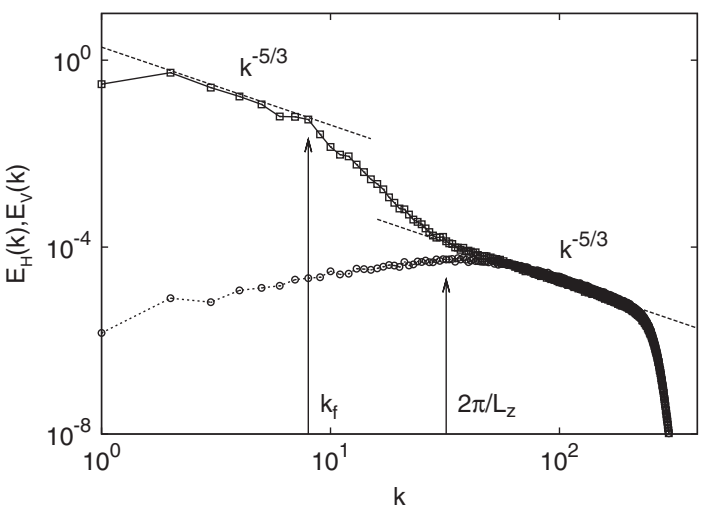

FIG. 3. Kinetic energy spectrum of horizontal (squares) and vertical (circles) velocities. Dashed lines represents Kolmogorov scaling. Parameters of the simulation: $L_{x}=2 \pi, \ell_{f} / L_{x}=1 / 8$, $L_{z} / L_{x}=1 / 32, \nu_{8}=10^{-37}$.

Yet, we cannot exclude, on the basis of present data, the possibility that a residual inverse cascade survives. Well designed experiments with electromagnetically driven thin fluid layers joint with high-resolution direct numerical simulations could provide the key to settle this fundamental question about the nature of turbulence.

The authors are grateful to Guido Boffetta and Sylvain Rubenthaler for fruitful discussions. Numerical simulations were performed at CINECA and on the INFN Turbofarm cluster in Turin, Italy.

[1] U. Frisch, Turbulence: The Legacy of A. N. Kolmogorov (Cambridge University Press, Cambridge, England, 1995).

[2] R. H. Kraichnan, Phys. Fluids 10, 1417 (1967).

[3] D. Bernard, G. Boffetta, A. Celani, and G. Falkovich, Nature Phys. 2, 124 (2006).

[4] J. D. Fournier and U. Frisch, Phys. Rev. A 17, 747 (1978).

[5] P. Giuliani, M. H. Jensen, and V. Yakhot, Phys. Rev. E 65, 036305 (2002).

[6] V.S. L'vov, A. Pomyalov, and I. Procaccia, Phys. Rev. Lett. 89, 064501 (2002).

[7] G. Falkovich, K. Gawędzki, and M. Vergassola, Rev. Mod. Phys. 73, 913 (2001).

[8] R. H. Kraichnan, Phys. Fluids 11, 945 (1968).

[9] M.I. Freidlin, Functional Integration and Partial Differential Equations (Princeton University Press, Princeton, NJ, 1985).

[10] K. Gawę̧dzki and M. Vergassola, Physica (Amsterdam) 138D, 63 (2000).

[11] A. Celani, S. Rubenthaler, and D. Vincenzi, J. Stat. Phys. 138, 579 (2010).

[12] K. Gawȩdzki and P. Horvai, J. Stat. Phys. 116, 1247 (2004).

[13] L. M. Smith, J. R. Chasnov, and F. Waleffe, Phys. Rev. Lett. 77, 2467 (1996); K. Ngan, D. N. Staub, and P. Bartello, Phys. Fluids 17, 125102 (2005). 